\title{
Morphological evidence for an invasion-independent metastasis pathway exists in multiple human cancers
}

\author{
Takashi Sugino*1, Tomiko Yamaguchi ${ }^{1}$, Go Ogura1, Atsuko Saito², \\ Takeaki Hashimoto ${ }^{1}$, Nobuo Hoshi ${ }^{1}$, Sayaka Yoshida ${ }^{3}$, Steve Goodison ${ }^{4}$ and \\ Toshimitsu Suzuki ${ }^{1}$
}

\begin{abstract}
Address: ${ }^{1}$ Department of Pathology, Fukushima Medical University School of Medicine, Fukushima, 960-1295, Japan, 2 Pathology Division, Jusendo General Hospital, Koriyama, 963-8002, Japan, ${ }^{3}$ Department of Surgery, Fukushima Medical University School of Medicine, Fukushima, 960-1295, Japan and ${ }^{4}$ Department of Pathology, University of Florida, Jacksonville, FL 32209-6511, USA

Email: Takashi Sugino* - sugino@fmu.ac.jp; Tomiko Yamaguchi - yamat@fmu.ac.jp; Go Ogura - g-ogura@fmu.ac.jp; Atsuko Saito - a.saito@jusendo.or.jp; Takeaki Hashimoto - thashi@fmu.ac.jp; Nobuo Hoshi - nobuo@fmu.ac.jp;

Sayaka Yoshida - ysayaka@fmu.ac.jp; Steve Goodison - steve.goodison@jax.ufl.edu; Toshimitsu Suzuki - tmt@fmu.ac.jp

* Corresponding author
\end{abstract}

This article is available from: http://www.biomedcentral.com/I74I-70I5/2/9

(C) 2004 Sugino et al; licensee BioMed Central Ltd. This is an Open Access article: verbatim copying and redistribution of this article are permitted in all media for any purpose, provided this notice is preserved along with the article's original URL.

\begin{abstract}
Background: We have previously described an alternative invasion-independent pathway of cancer metastasis in a murine mammary tumor model. This pathway is initiated by intravasation of tumor nests enveloped by endothelial cells of sinusoidal vasculature within the tumor. In this study, we examined whether evidence for the invasion-independent pathway of metastasis is present in human cancers.
\end{abstract}

Methods: Archival specimens of 10 common types of human cancers were examined for the presence of sinusoidal vasculature enveloping tumor nests and subsequently generated endothelialcovered tumor emboli in efferent veins.

Results: A percentage of tumor emboli in all cancers was found to be enveloped by endothelial cells, but these structures were particularly prevalent in renal cell carcinomas, hepatocellular carcinomas and follicular thyroid carcinomas. A common feature of the vasculature in these tumors was the presence of dilated sinusoid-like structures surrounding tumor nests. A high mean vascular area within tumors, an indication of sinusoidal vascular development, was significantly related to the presence of endothelial-covered tumor emboli.

Conclusions: These results suggest that an invasion-independent metastatic pathway is possible in a wide variety of human cancers. Further investigation of this phenomenon may present new therapeutic strategies for the amelioration of cancer metastasis.

\section{Background}

Cancer metastasis is most often described as a series of sequential processes that involve the following steps: growth of new blood vessels into the primary tumor, local invasion of the extracellular matrix, intravasation through proteolysis of components of the blood vessel wall and survival during transport in the bloodstream. After reaching the target organ, adhesion to endothelial lining occurs, followed by extravasation through the vessel wall and subsequent proliferation at the secondary site. 
Accordingly, it is widely believed that active invasion by cancer cells is essential to the metastatic process $[1,2]$.

However, we have recently reported a murine mammary tumor model for blood-borne metastasis that does not require invasion of the vascular wall at either the primary tumor or the target organ $[3,4]$. The process involves intravasation of tumor nests that are surrounded by blood vessels, transportation of tumor emboli enveloped with endothelial cells and intravascular tumor growth in the lung without penetration of the vascular wall at the secondary site. Our comparative studies between highly metastatic and non-metastatic cells suggest that tumor cell intravasation, induced by high angiogenic activity and sinusoidal remodeling of tumor blood vessels, is a key step in the invasion-independent metastatic pathway $[3,4]$.

Microscopic observations of routine surgical specimens suggest that the invasion-independent pathway may occur in some types of human cancers. For example, intravascular tumor emboli from follicular carcinoma of the thyroid have been observed as being enveloped with endothelial cells $[5,6]$. However, there has not been any report describing the meaning of this phenomenon in the metastatic process. Moreover, little attention has been paid to the existence of tumor embolus-associated endothelia in other human cancers.

The aim of this study was to take the findings we observed in our murine model of metastasis [4] and to evaluate whether evidence for an invasion-independent metastatic pathway exists in human cancers. Interestingly, analysis of archival tissue samples obtained from several types of cancers did reveal the presence of endothelium-coated tumor cell emboli in primary tumor vasculature. Furthermore, the immunochemical assessment of tumor vasculature identified a positive correlation between the status of sinusoidal vasculature and the presence of endotheliumcoated tumor emboli, indicating a link between tumor angiogenesis and the facilitation of an invasion-independent metastatic pathway.

\section{Methods}

\section{Tissue procurement}

An immunohistochemical study was performed using formalin-fixed, paraffin-embedded tissue specimens obtained from the collections of Fukushima Medical University and Jusendo General Hospital. Ten types of human cancers were chosen for examination. Primary tumor specimens originated from the following organ systems: thyroid, liver, kidney, stomach, colon, breast, pancreas, lung, uterus and esophagus. Ten cases from each cancer type were selected for immunohistochemical analysis. Samples that had tumor emboli present in the afferent veins were selected. Archival tissues and anonymized data were used in accordance with national and local human material investigative protocols.

\section{Immunohistochemistry}

Monoclonal antibodies to CD34 (clone QBEND10; Immunotech, Hamburg, Germany) and CD31 (clone JC70; Dako, Grostrup, Denmark) were used for endothelial cell staining on $3-\mu \mathrm{m}$ paraffin-embedded sections according to the manufacturer's instructions. Immunohistochemical staining was performed using an indirect streptavidin-biotin immunoperoxidase method (SAB-PO (M) kit, Nichirei Corp., Tokyo, Japan). After blocking of endogenous peroxidase activity in $0.3 \%$ hydrogen peroxide in methanol for $30 \mathrm{~min}$, slides were incubated with primary antibodies overnight at $4^{\circ} \mathrm{C}$, washed with PBS, and then incubated with secondary biotin-labeled antibodies for $30 \mathrm{~min}$ at room temperature. Antibody localization was visualized with peroxidase-conjugated streptavidin for $30 \mathrm{~min}$ at room temperature, followed by the diaminobenzidene reaction. The slides were counterstained with hematoxylin.

\section{Assessment of tumor vascularity}

Tumor vascularity was assessed using two parameters. Microvessel density was measured according to standard procedures [7]. Briefly, immunochemically-stained slides were first observed at low power magnification $(\times 100)$ to identify areas with the highest density of microvessels. In each case, the three most vascularized areas were selected, and microvessels in these areas were counted at high power magnification $(\times 200)$ in a grid area of $0.19 \mathrm{~mm}^{2}$. Three areas of high vascular density were counted on each section, and the vascular density was determined. The second parameter assessed was the mean area of tumor blood vessels. The vascular area was evaluated in three low-power fields $(\times 40)$ on each case. The areas of microvessels (exclusive of arteries and veins) were measured in each field using a computerized image analyzer (ImagePro Plus, Media Cybernetics, Silver Spring, Maryland, USA). The percentage of total vascular area in each tumor parenchyma was determined.

\section{Statistical analysis}

The student's $t$ test was used to compare groups of mean values of vascular density and percentage of vascular area; $P<0.01$ was considered statistically significant.

\section{Results \\ A wide variety of human cancers generate tumor emboli which become enveloped with endothelial cells}

Results compiled from the analysis of 10 cases per cancer type, and of 10 different types of human cancers, are listed in Table 1. Intravascular tumor emboli were categorized as two groups: emboli covered with endothelial cells and 
Table I: Endothelial covering on intravascular tumor emboli from human cancers.

\begin{tabular}{lll}
\hline Origin of cancer & $\begin{array}{l}\text { Histological type } \\
\text { (number of cases) }\end{array}$ & Endothelial covering \\
\hline Thyroid & FTC (10) & 10 \\
Liver & HCC (10) & 10 \\
Kidney & RCC (10) & 9 \\
Stomach & ADC (10) & 1 \\
Colon & ADC (10) & 2 \\
Breast & ADC (10) & 3 \\
Pancreas & ADC (10) & 1 \\
Lung & SQCC (4), ADC (6) & 2 \\
Uterus & SQCC (5), ADC (5) & 1 \\
Esophagus & SQCC (10) & 1
\end{tabular}

FTC, follicular thyroid carcinoma; HCC, hepatocellular carcinoma; RCC, renal cell carcinoma; ADC, adenocarcinoma; SQCC, squamous cell carcinoma.

naked emboli with no evident endothelial association. The majority of intravascular tumor emboli present in all cases of renal cell carcinoma (RCC) (Figure 1A), hepatocellular carcinoma (HCC) (Figure 1B) and follicular thyroid carcinoma (FTC) (Figure 1C) were coated with a CD31, and CD34-positive endothelial lining. The vasculature of these three types of cancers formed well-developed sinusoidal structures enveloping tumor nests (Figure 1, AIII, BIII, CIII). These tumors exhibited an intravasation feature in which tumor nests enveloped with sinusoidal vessels entered the collecting drainage vein. Most of the tumor emboli present in the other seven types of human cancers were naked, homogeneous structures with no endothelial covering evident (Figure 2A). Many of those with naked emboli contained small veins filled with organized fibrous tissue coating the tumor emboli, perhaps due to vascular injury or tumor-associated thrombosis. However, in all types of cancers tested, there were cases with tumor emboli that were directly covered with endothelial cells entirely (Figure 2B), or partially (Figure 2C), and with only scanty intervening fibrous tissue evident. The tumors possessed a well-developed sinusoidal vasculature surrounding and enveloping the tumor nests (Figure 2D).

\section{Sinusoidal vascular development may facilitate intravasation of tumor cells in an invasion-independent manner}

In order to assess accurately tumor vascularity, we measured both vascular density (Figure $3 \mathrm{~A}, 3 \mathrm{~B}$ ) and the percentage of vascular area (Figure 3C,3D) in the primary tumors. Both the median mean vascular density and the percentage of vascular area were significantly greater in the cases that exhibited endothelium-coated tumor emboli (Figure 3A,3C). Vascular densities in FTC and RCC samples were much greater than in any other cancers. HCC samples did not show high vascular density in spite of having well-developed vasculature (Figure 3B). Conversely, the percentage of vascular area in all three cancers developing sinusoidal vasculature (FTC, HCC and RCC) was significantly higher than that observed in the other seven cancer types (Figure 3D). Moreover, in seven cancer types, exclusive of FTC, HCC and RCC which have welldeveloped sinusoidal vasculature in most cases, the median mean percentage of vascular area was also significantly higher in cases with endothelial-coated tumor emboli $(P<0.01)$, whereas the mean vascular density did not detect the difference $(P=0.06)$.

\section{Discussion}

The invasive property of cancer cells is generally believed to be one of the most essential factors in the multi-step process of metastasis, enabling the metastatic cells to penetrate the vascular wall barrier at either the primary or secondary site of growth $[8,9]$. However, we have previously proposed an alternative metastatic model whereby tumors gain access to the host vasculature in a mechanism independent of active invasion. We developed a murine model of mammary tumor metastasis (MCH66), in which tumor cell nests became surrounded by sinusoidal blood vessels and entered the circulation as endothelium-coated tumor cell emboli $[3,4]$. The isolation from our murine model of monoclonal cell lines that have differential propensities to achieve this mode of intravasation, indicates that there is a tumor cell-specific genetic component to this phenomenon. In the present study, we examined whether a similar phenomenon exists in human cancers by monitoring the association of endothelia with intravascular tumor emboli and sinusoidal development of tumor vasculature enveloping tumor nests - major features of the invasion-independent metastasis pathway. Indeed, cases with both indices were detected in all cancer types examined, but with particularly high incidence in renal cell carcinoma, follicular thyroid carcinoma and hepatocellular carcinoma.

Endothelial cell association with tumor emboli in FTC has been described previously $[5,6,10]$, but neither the mechanism nor clinical significance of this phenomenon has been investigated. To our knowledge, there have been no reports describing whether tumor emboli from HCC, RCC or any other cancers are consistently associated with vascular endothelial cells.

The observed correlation of the morphology of tumor vasculature with the presence of endothelium-coated tumor emboli in this study suggests a possible mechanism for an alternative pathway of tumor cell dissemination and metastasis. Such a mechanism is depicted in Figure 4 and has been previously proposed and discussed by ourselves 


\section{A: Renal cell carcinoma}
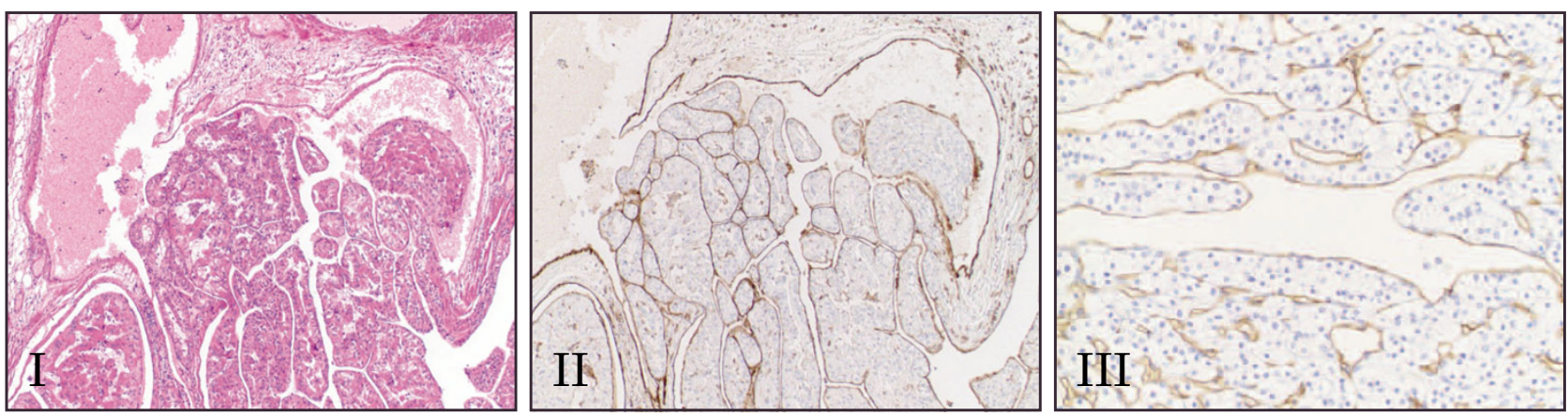

\section{B: Hepatocellular carcinoma}
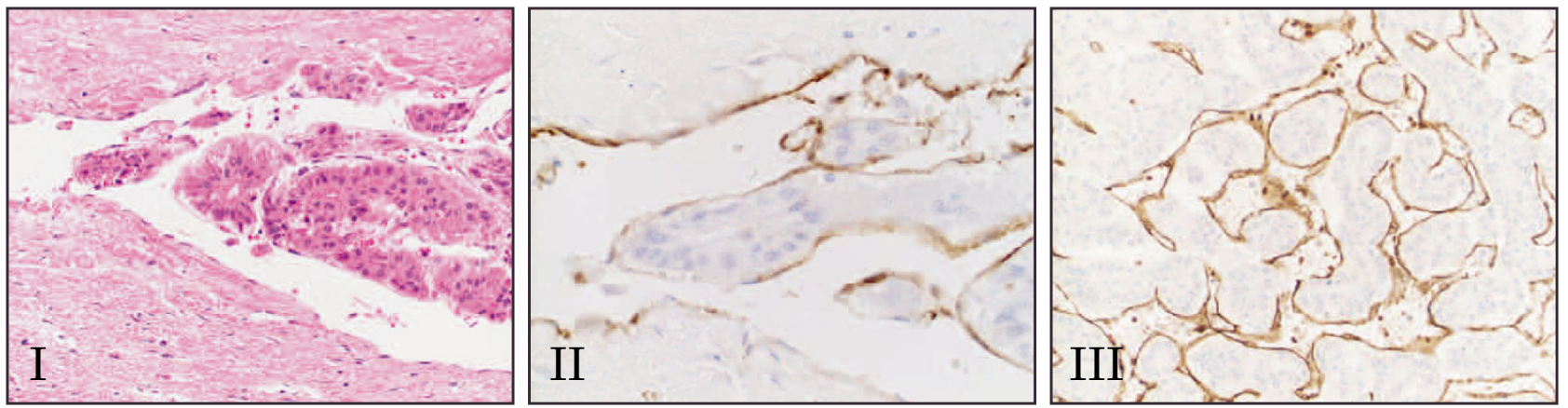

\section{C: Follicular thyroid carcinoma}
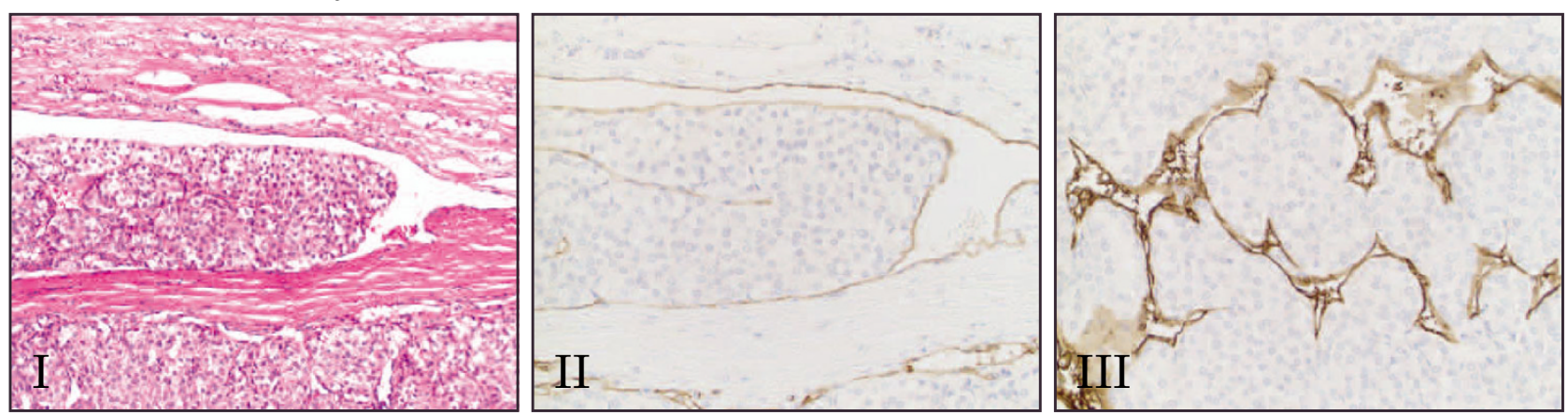

Figure I

Representative photomicrographs of primary tumor vasculature and examples of tumor emboli within efferent veins. (A) Renal cell carcinoma, (B) hepatocellular carcinoma and (C) follicular thyroid carcinoma. An endothelial layer covering intravascular tumor emboli can be seen using immunostaining with anti-CD3I antibody (AII, BII, CII). The vasculature in the primary tumors of the three carcinomas formed sinusoidal structures surrounding tumor cell nests (anti-CD3I staining, Alll, BIII, CIII). $\mathrm{Al}, \mathrm{BI}$ and $\mathrm{Cl}$ are $\mathrm{H} \& \mathrm{E}$ staining. Original magnifications: (AII, BII, CII) $\times 400$, all others $\times 200$. 

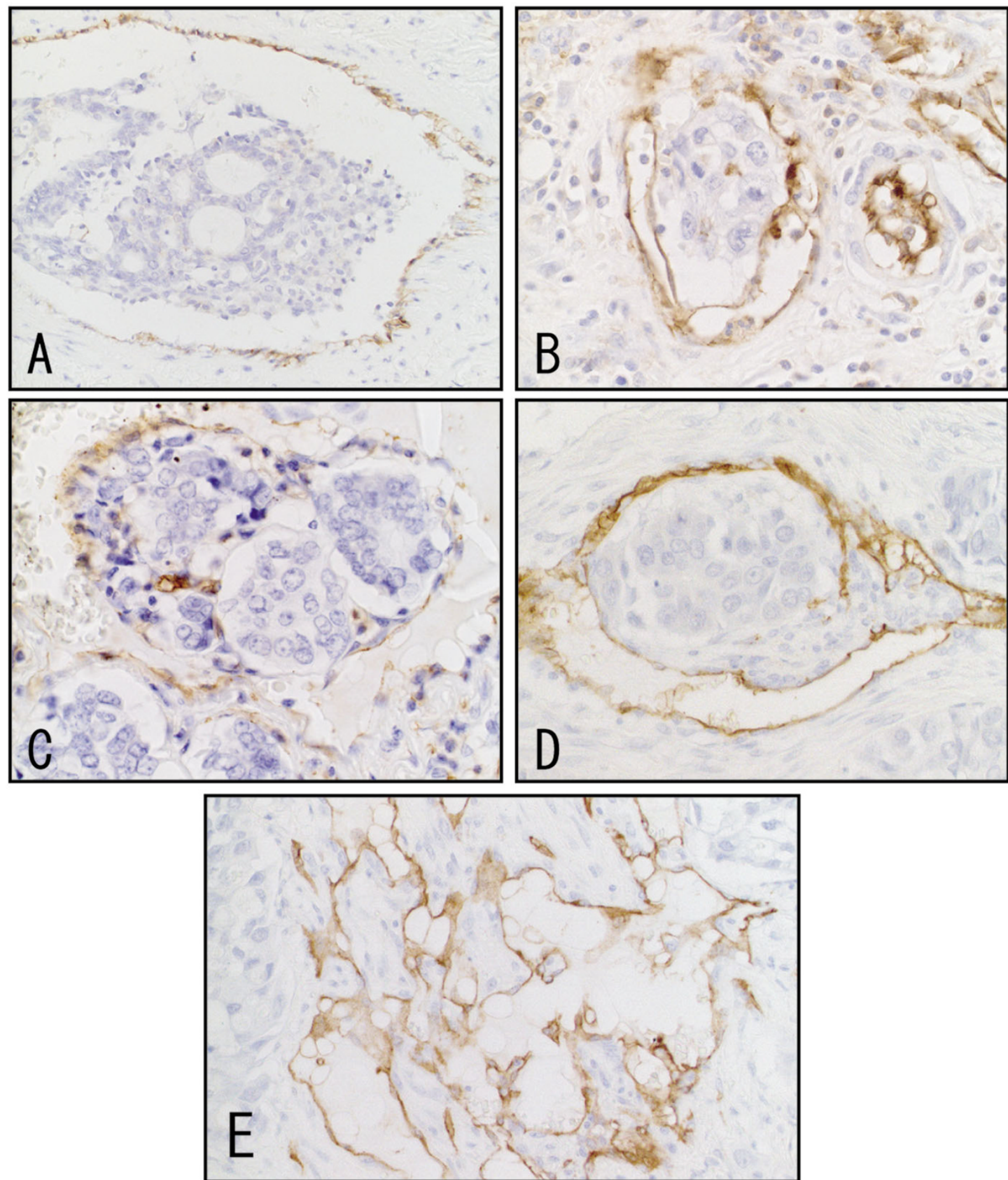

Figure 2

Representative photomicrographs of intravascular tumor. Endothelial cells were detected and visualized using immunostaining with $(\mathbf{A}, \mathbf{C})$ anti-CD3I and (B, D) anti-CD34 antibodies. (A) An example of a naked tumor embolus, that is, no evident endothelial covering, within an efferent vein in a case of breast carcinoma. (B) In a case of esophageal carcinoma, a tumor embolus within a vein is entirely covered with endothelial cells. (C, D) Tumor emboli originating from a breast carcinoma are enveloped by endothelial cells. (E) The same case as D. The tumor possessed well-developed sinusoidal vasculature. Original magnifications: $(A, E), \times 200 ;(B-D), \times 400$. 

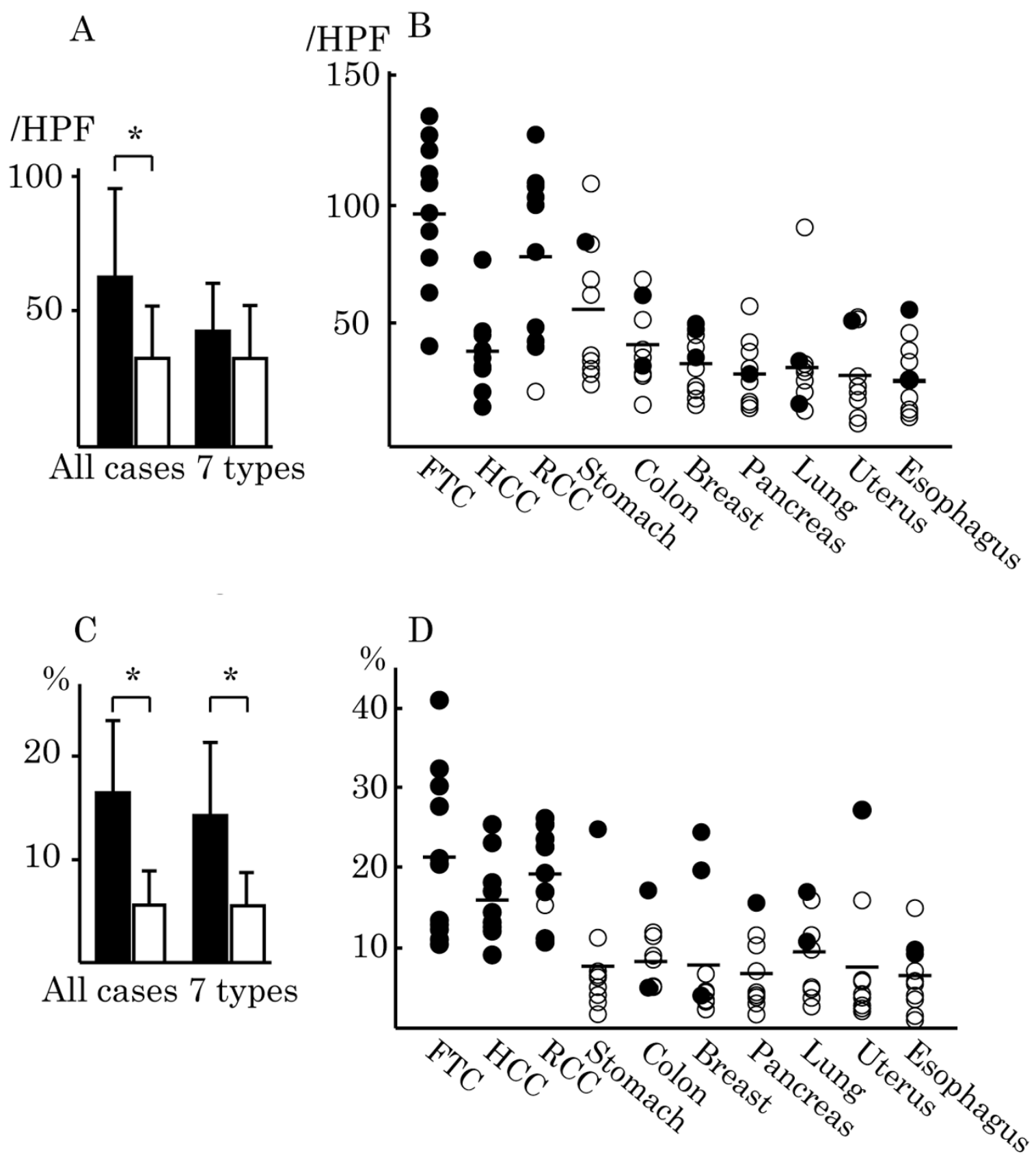

Figure 3

Tumor vascularity in cancer cases with and without evidence of the presence of endothelium-coated tumor emboli. Vascular density was determined by counting the number of vessels per unit of HPF (high power field) at magnification $\times 400$. (A) Mean vascular density of total cases and seven cancer types, excluding FTC, HCC and RCC. Standard deviation (SD) is indicated by error bars. (B) Vascular density of individual cases for each type of cancer. The mean vascular densities are indicated by the horizontal lines. (C) Mean percentage of vascular area and SD of all cases examined and for the seven cancer types. (D) Percentage of vascular area of individual cases for each type of cancer. The mean percentages of vascular area are indicated by the horizontal lines. Solid bars and circles, cancer cases with endothelium-coated tumor emboli; open bars and circles, cases with uncoated emboli. FTC, follicular thyroid carcinoma; HCC, hepatocellular carcinoma; RCC, renal cell carcinoma. *Significant difference between cancer cases with endothelium-coated tumor emboli and with naked ones $(P<0.0 \mathrm{I})$. 


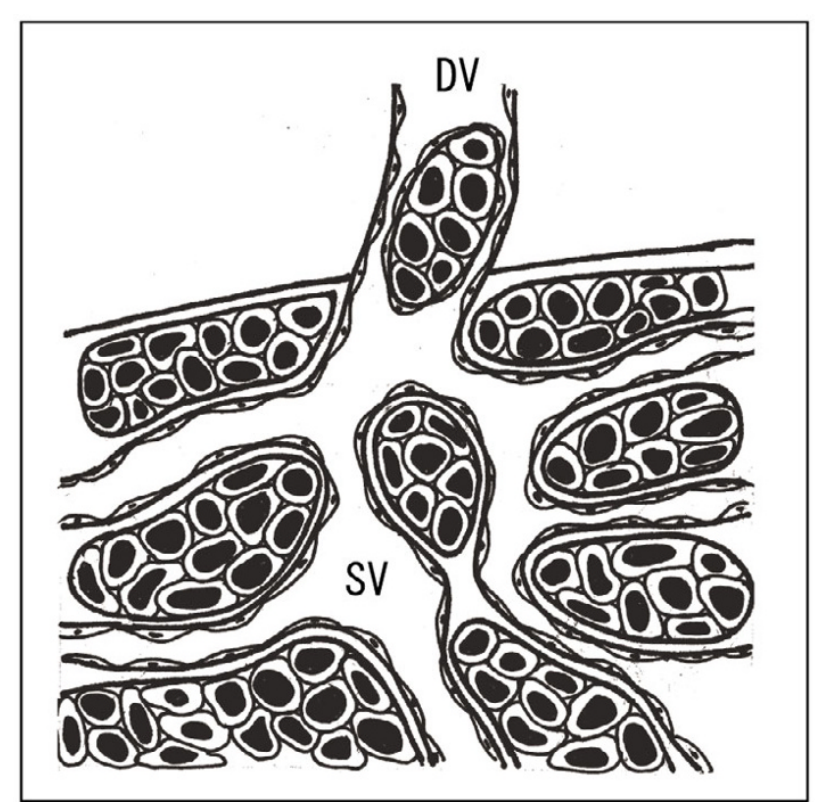

Figure 4

Schematic representation of an invasion-independent pathway of intravasation by human cancer cells. Tumor cell nests become surrounded by sinusoidal blood vessels (SV) and enter the collecting drainage vein (DV) as endothelial-coated tumor cell emboli.

and others $[4,11]$. A common feature of the vasculature in the primary tumors carrying endothelium-coated tumor emboli was the presence of sinusoid structures surrounding tumor nests. In fact, FTC, RCC and HCC are known as hypervascular tumors, that is, harboring large vascular channels which have the appearance of sinusoidal capillaries [12-14]. In these cancers, re-organized tissue structures consisting of tumor nests and surrounding sinusoidal vessels presumably become the embolus 'unit', which is subsequently disseminated. Therefore, this pathway may depend not on tissue destruction by cancer cell invasiveness but on remodeling of tissue architecture through tumor-stroma interactions.

Our results indicate that an invasion-independent metastasis pathway is related to both angiogenesis and vascular remodeling. Microvessel density is assumed to reflect the level of tumor angiogenesis. Many studies have reported a correlation of vascular density with the occurrence of metastasis and poor prognosis [15-19]. In this study, a positive correlation of mean vascular density with the presence of endothelium-coated tumor emboli suggests that angiogenic factors may be responsible for inducing this metastatic pathway. In contrast to the mean value of groups of cancer cases, vascular density in individual cases, especially of HCC that is reportedly hypervascular, did not necessarily reflect their sinusoidal vascular development, or the endothelial association with the accompanying tumor emboli. The reason for this may be the often peculiar vascular structure, consisting of dilated and fused sinusoidal vessels, which prove difficult to quantify accurately. Instead of vascular density, measurements of vascular area or microvessel fractal dimension are used to quantify intratumoral vascularity, especially for the assessment of dilated and complex vasculature such as sinusoidal vessels in FTC and RCC $[13,14,20]$. In this study, intratumoral vascular area was more tightly correlated than vascular density in individual cases with endothelium-coated tumor emboli. Our data demonstrate that the invasion-independent metastatic pathway can be controlled not only by increasing the number of blood vessels attracted to the tumor, but also by vascular remodeling into a more sinusoidal structure.

\section{Conclusions}

Our analyses demonstrate that conditions for an invasionindependent dissemination pathway exist in a wide variety of human cancers. Our analysis of morphology and vascularity suggest that this metastatic pathway can be initiated by the development of sinusoidal tumor vasculature. It is also possible that the alternative dissemination pathway leads to an improved metastatic efficiency. The defensive morphology of endothelium-associated tumor emboli may protect the tumor cells from hemodynamic forces and from immune surveillance systems. Furthermore, as observed in our mammary metastasis model [4], proliferation in a distant secondary site may be facilitated by the pre-requisite intimate association with stromal components. Further studies analyzing the molecular mechanisms of this type of dissemination, and the clinico-pathological and prognostic significance of an invasion-independent metastasis pathway will probably lead to the development of new treatment agents and strategies for cancer metastasis.

\section{Competing interests}

None declared.

\section{Authors' contributions}

TS conceived the study, participated in its design and coordination, and drafted the manuscript. TY assisted with the immunohistochemical staining. GO, AS, TH, $\mathrm{NH}, \mathrm{SY}, \mathrm{SG}$ and TS participated in study design and evaluations, critical discussion and manuscript preparation. All authors read and approved the final manuscript.

\section{Acknowledgements}

This work was supported in part by a Grant-in-Aid for Scientific Research (C) (no. 14570 126) from the Ministry of Education, Science, Sports and Culture, Japan. We thank Natsuko Takahashi for excellent technical assistance. 


\section{References}

I. Fidler IJ, Balch CM: The biology of cancer metastasis and implications for therapy. Curr Probl Surg 1987, 24:129-209.

2. Chambers AF, Groom AC, MacDonald IC: Dissemination and growth of cancer cells in metastatic sites. Nat Rev Cancer 2002, 2:563-572.

3. Sugino T, Kawaguchi T, Suzuki T: Sequential process of bloodborne lung metastases of spontaneous mammary carcinoma in C3H mice. Int J Cancer 1993, 55: I4 I- I47.

4. Sugino T, Kusakabe T, Hoshi N, Yamaguchi T, Kawaguchi T, Goodison S, Sekimata M, Homma Y, Suzuki T: An invasion-independent pathway of blood-borne metastasis: a new murine mammary tumor model. Am J Pathol 2002, 160:1973-1980.

5. Rosai J, Carcangui ML, DeLellis RA: Tumors of the thyroid gland. Atlas of Tumor Pathology. Edited by: Rosai J and Sobin L H. Washington DC, Armed Forces Institute of Pathology; 1992:49-63.

6. Thompson LD, Wieneke JA, Paal E, Frommelt RA, Adair CF, Heffess CS: A clinicopathologic study of minimally invasive follicular carcinoma of the thyroid gland with a review of the English literature. Cancer 200I, $91: 505-524$

7. Fox SB: Microscopic assessment of angiogenesis in tumors. Methods in Molecular Medicine: Angiogenesis Protocols Volume 46. Edited by: Murray J C. Totowa, NJ, Humana Press, Inc.; 200I:29-46.

8. Liotta LA, Tryggvason K, Garbisa S, Hart I, Foltz CM, Shafie S: Metastatic potential correlates with enzymatic degradation of basement membrane collagen. Nature 1980, 284:67-68

9. Egeblad M, Werb Z: New functions for the matrix metalloproteinases in cancer progression. Nat Rev Cancer 2002, 2: 16I- 174.

10. Franssila KO, Ackerman LV, Brown CL, Hedinger CE: Follicular carcinoma. Semin Diagn Pathol 1985, 2: I0I-122.

II. Nakashima T, Kojiro M, Kawano Y, Shirai F, Takemoto N, Tomimatsu Y, Kawasaki H, Okuda K: Histologic growth pattern of hepatocellular carcinoma: relationship to orcein (hepatitis B surface antigen)-positive cells in cancer tissue. Hum Pathol 1982, 13:563-568

12. Sabo E, Boltenko A, Sova Y, Stein A, Kleinhaus S, Resnick MB: Microscopic analysis and significance of vascular architectura complexity in renal cell carcinoma. Clin Cancer Res 200I, 7:533-537.

13. Wong NA, Willott J, Kendall MJ, Sheffield EA: Measurement of vascularity as a diagnostic and prognostic tool for well differentiated thyroid tumours: comparison of different methods of assessing vascularity. J Clin Pathol 1999, 52:593-597.

14. Macchiarini P, Fontanini G, Hardin MJ, Squartini F, Angeletti CA: Relation of neovascularisation to metastasis of non-smallcell lung cancer. Lancet 1992, 340: | 45- I 46.

15. Gasparini G: Prognostic and predictive value of intra-tumoral microvessel density in human solid tumours. Tumour angiogenesis Edited by: Bicknell R, Lewis C E and Ferrara N. Oxford, U. K Oxford Univ. Press; 1997:29-44.

16. Weidner N: Tumoral vascularity: What does it tell us about the growth and spread of cancer? Tumor angiogenesis and microcirculation Edited by: Voest E E and D'Amore P A. New York, U. S. A., Marcel Dekker, Inc.; 200 I:465-486.

17. Weidner N, Folkman J, Pozza F, Bevilacqua P, Allred EN, Moore DH Meli S, Gasparini G: Tumor angiogenesis: a new significant and independent prognostic indicator in early-stage breast carcinoma. J Nat/ Cancer Inst 1992, 84: I875- I887.

18. Bochner BH, Cote RJ, Weidner N, Groshen S, Chen SC, Skinner DG, Nichols PW: Angiogenesis in bladder cancer: relationship between microvessel density and tumor prognosis. J Natl Cancer Inst 1995, 87: 1603-1612.

19. Pavlopoulos PM, Konstantinidou AE, Agapitos E, Kavantzas N, Nikolopoulou P, Davaris P: A morphometric study of neovascularization in colorectal carcinoma. Cancer 1998, 83:2067-2075.

20. Simpson JF, Ahn C, Battifora H, Esteban JM: Endothelial area as a prognostic indicator for invasive breast carcinoma. Cancer 1996, 77:2077-2085.

\section{Pre-publication history}

The pre-publication history for this paper can be accessed here:

http://www.biomedcentral.com/1741-7015/2/9/prepub
Publish with Bio Med Central and every scientist can read your work free of charge

"BioMed Central will be the most significant development for disseminating the results of biomedical research in our lifetime. "

Sir Paul Nurse, Cancer Research UK

Your research papers will be:

- available free of charge to the entire biomedical community

- peer reviewed and published immediately upon acceptance

- cited in PubMed and archived on PubMed Central

- yours - you keep the copyright
BioMedcentral 